\title{
NDT Inspection of Aeronautical Components by Projected Thermal Diffusivity Analysis
}

\author{
by P. Venegas***, J. Perán**, R. Usamentiaga***, I. Sáez de Ocáriz* \\ * Aeronautical Technologies Centre, Juan de la Cierva 1, 01510 Miñano, Spain, pablo.venegas@ctaero.com, \\ idurre.saezdeocariz@ctaero.com \\ ** National Distance Education University, Department of Applied Mathematics, C/ Juan del Rosal 12, 28040 \\ Madrid, Spain, jperan@ind.uned.es

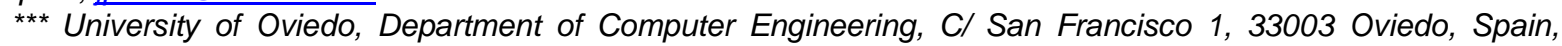 \\ rusamentiaga@uniovi.es
}

\begin{abstract}
This paper analyses the application of an innovative method for thermographic NDT data processing to inspections on real aeronautical components. The results provided by this method are related to thermal diffusivity values obtained by projecting the characteristics of a 3D thermal diffusion model onto one of the coordinate planes. In previous studies, laboratory experiments demonstrated that this method produces a higher increase in SNR compared to conventional processing algorithms and it also provides a novel way of representing detected defects identifying the area affected by the lateral thermal diffusion effect.
\end{abstract}

\section{Introduction}

Infrared thermography (IRT) is becoming a well-known technology due to its broad application range and special features. The operating principle consists basically in the relation between the infrared radiation emitted by objects and their temperature, and other parameters involved in physical processes linked to heat flow phenomena. The application of IRT for nondestructive testing (NDT) of materials is spreading quickly [1]. The rapidity and cleaning of the thermographic NDT technique make it suitable for inspecting materials in different industrial processes, especially in those industries where the components are thin, such as aeronautics [2].

The IRT NDT inspection is based on the application of a thermal stimulation to the material to be inspected and the subsequent analysis of the induced thermal evolution. Different stimulation methods may be applied in order to generate the necessary heat flow that enables the detection of anomalies in a material. The raw data captured by the IR sensor is normally used as temperature control method [3]. However, this raw data is not directly used for defect identification. Except in special cases where the detection is evident, the thermal anomalies produced by internal defects are so subtle that the signal is lost in the thermographic data noise and the defects remain undetected. The common procedure for data analysis includes a processing stage of the raw data to enhance the defects response and reduce the noise level. There exist many different mathematical algorithms to process IRT NDT data [4,5]. Each processing algorithm provides different benefits and is usually more suitable for specific type of materials, defects or heat flow conditions. They take the temperature raw data as input and produce output signals that depend on the processing procedure. Common outputs are amplitude or phase of complex data, time derivatives and statistical parameters among others.

Projected thermal diffusivity (PTD) analysis is a processing algorithm recently developed [6] that operates with temperature values as input data to produce an output related to diffusivity values. This algorithm has been analysed at laboratory level using specimens with intentionally induced defects. The results obtained were satisfactory with high detectability levels. The application of this algorithm to the inspection on real components is the next step in the evaluation process. This paper shows the results obtained in a preliminary inspection campaign on real aeronautical parts analysed with the PTD method.

\section{Projected thermal diffusivity method}

\subsection{Infrared thermographic NDT modelling}

An IRT NDT inspection may be modelled by the thermal diffusion differential equation, which takes into account the specific test conditions and material properties involved in the process. Assuming that several simplifying hypotheses are met, such as the fact that the material under inspections is opaque, heterogeneous and isotropic, no chemical reaction occurs as a consequence of the heat irradiation, no internal heat sources exist inside the material, the thermophysical properties of the medium remain constant during the inspection and are independent of temperature, the external surface behaves as a grey body and the incident energy is diffuse and homogeneously distributed along the surface, the thermal process that takes place in IRT NDT inspections may be properly represented by the heat diffusion 

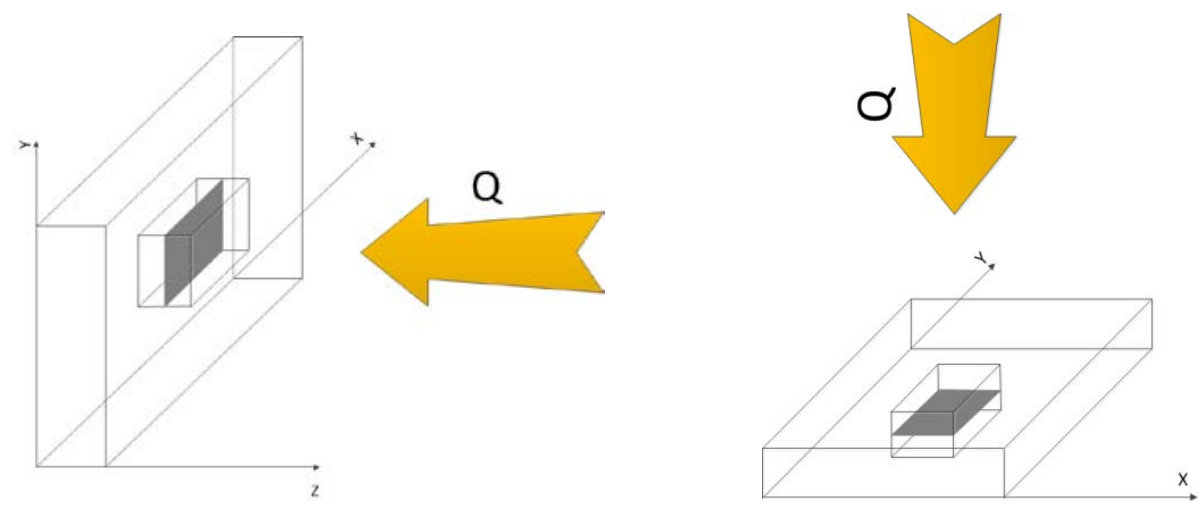

Fig. 1. $3 D$ thermal diffusion model (left) and $2 D$ simplified thermal diffusion model (right).

equation (1), where $u$ is the temperature at each material point, $c$ is the thermal diffusivity of the material $\left(c=k /\left(\rho c_{p}\right)\right), k$ is the thermal conductivity, $\rho$ is the material density, $c_{p}$ is the specific heat at constant pressure and $\nabla$ is the nabla operator with respect to the spatial variables, and $F$ is the heat sources.

$$
\begin{aligned}
& \frac{\partial u(x, t)}{\partial t}-\nabla \cdot(c(x) \nabla u(x, t))=F(x, t) \\
& x \in \mathfrak{R}^{3}, t \geq 0, c(x) \in \mathfrak{R}^{+}
\end{aligned}
$$

The thermal evolution of the object under inspection is then obtained by solving this differential equation, producing information that may be used for determining important test parameters, e.g. the minimum thermal contrast produced by certain type of defect, the amount of energy necessary to be supplied to make a defect detectable by a IR sensor or the maximum depth possible to stimulate under certain heating conditions. The usefulness of the heat differential equation for IRT NDT analysis is demonstrated, and nowadays many researchers and inspectors use it as an evaluation stage previously to the experimental inspections. However, since the prior knowledge of material properties and stimulation characteristics is necessary, the use of this equation is limited to theoretical analysis.

\subsection{Derivation of the projected thermal diffusivity method}

The projected thermal diffusivity method (PTD) is developed with the aim of applying the thermal diffusivity equation to experimental tests [6]. The 3D thermal diffusion model cannot be directly used for this experimental purpose because the internal material temperature and diffusivity data remain unknown during the whole inspection. An alternative model is proposed to take advantage of the thermal equation in practical conditions. This is a 2D anisotropic diffusion model that is a simplified version obtained from the 3D heterogeneous isotropic model by projecting the characteristics onto one of the coordinate planes (figure 1). The physical parameters under study do not change with respect to the 3D case, but the domain of study and analysis becomes a surface in this simplified situation, reducing the number of existing unknowns in the problem.

This simplified model is mathematically formulated by the following equation:

$$
\begin{aligned}
& \frac{\partial u(x, t)}{\partial t}-\nabla \cdot(C(x, t) \nabla u(x, t))=F(x, t) \\
& x \in \mathfrak{R}^{2}, t \geq 0
\end{aligned}
$$

where $C(x, t)$ is the projected thermal diffusivity matrix, which consists in a $2 \times 2$ positive definite matrix with a general form:

$$
\left(\begin{array}{ll}
c_{11}(x, t) & c_{12}(x, t) \\
c_{21}(x, t) & c_{22}(x, t)
\end{array}\right)
$$

The most important difference between the 3D model and the simplified 2D model is the matrix $C(x, t)$. This matrix is a parameter related to the thermal diffusivity of the material. Unlike $c(x)$ in the 3D case, $c(x, t)$ has a surface definition domain and is able to capture information of near defects located at any depth so the deeper a defect is located the higher delay will experience the progress of the corresponding $C(x, t)$ values. Additionally, it must be considered that the physical meaning of the diffusivity values inside the matrix $C(x, t)$ may be completely lost as a result of the projection process. 


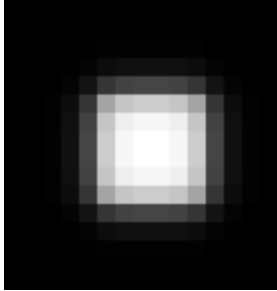

(a)

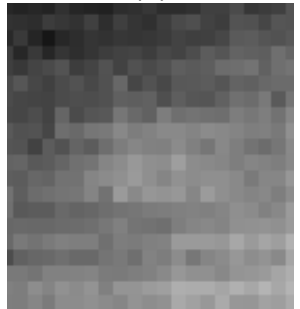

(d)

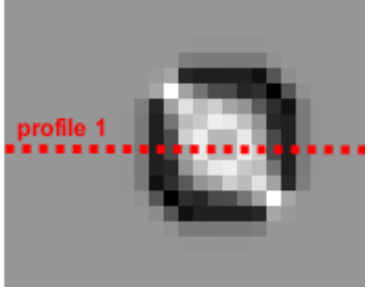

(b)

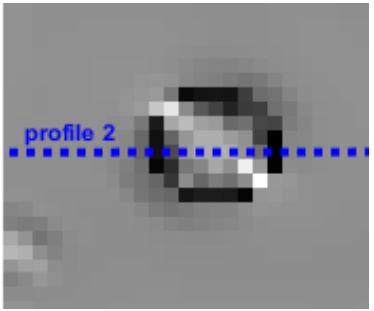

(e)

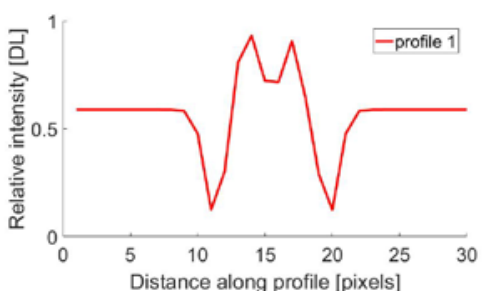

(c)

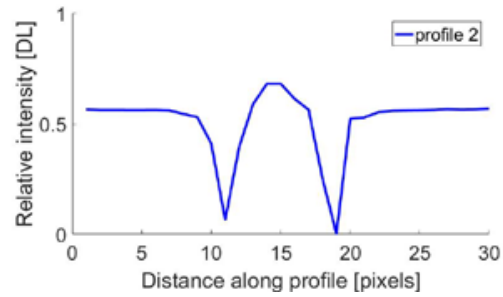

(f)

Fig. 2. Results obtained in computationally generated tests $(a, b, c)$ and results obtained in tests with calibrated samples $(d, e, f)$. Raw data $(a, d)$, PTD analysis $(b, e)$ and spatial profile of PTD analysis $(c, f)$.

Different situations may exist depending on the internal structure and health state of the object under inspection. Most cases can be classified in one of the following categories:

1. Infinite thickness zones without presence of defects

$$
\left(\begin{array}{cc}
c_{0} & 0 \\
0 & c_{0}
\end{array}\right)
$$

2. Finite thickness without presence of defects

$$
\left(\begin{array}{cc}
c_{0}(x, t) & 0 \\
0 & c_{0}(x, t)
\end{array}\right)
$$

3. Any thickness with presence of defects

$$
\left(\begin{array}{ll}
c_{11}(x, t) & c_{12}(x, t) \\
c_{21}(x, t) & c_{22}(x, t)
\end{array}\right)
$$

The projected thermal diffusivity method is derived from the previously developed anisotropic 2D diffusion equation (2) by the application of a discretization process. Finally, the algorithm to implement in the inspection procedure is expressed in matrix form as:

$$
\left[P_{i j}\right]=[T] C_{0}
$$

where $P_{i j}$ contains data related to the heating source, $[T]$ is a matrix made of known data parameters calculated from the input temperatures and $C_{0}$ is the unknown data set to be solved. The application of this result to the tests conducted in IRT NDT inspections needs the temperature values measured by the IR sensor as input data and provides the projected diffusivity values as output results.

\section{Results and discussion}

\subsection{Results in controlled samples}

The projected thermal diffusivity method has already been analysed with theoretical data computationally produced as well as with experimental data produced by thermographic inspections conducted on samples with calibrated defects [6]. The results obtained in both cases were satisfactory producing an increase in the defect detection level and providing a representation of results where areas with defects and near areas affected by lateral heat flow are clearly differentiated (figure 2). 


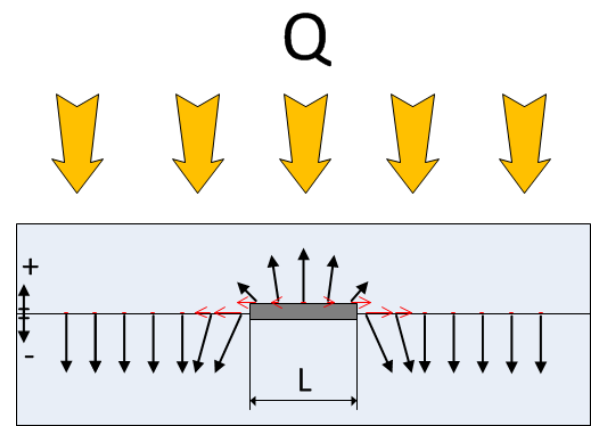

Fig. 3. Physical interpretation of the projected diffusivity values $C(x, t)$ obtained by the PTD method.

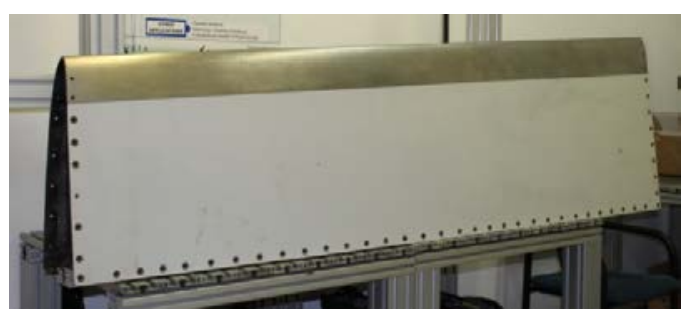

(a)

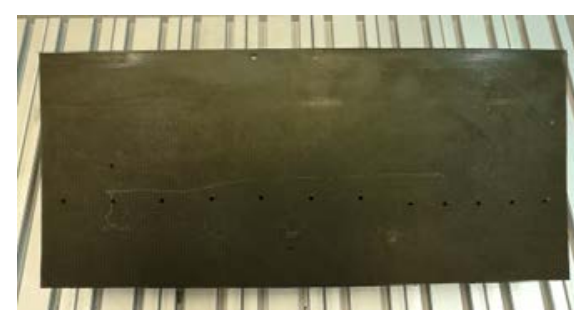

(b)

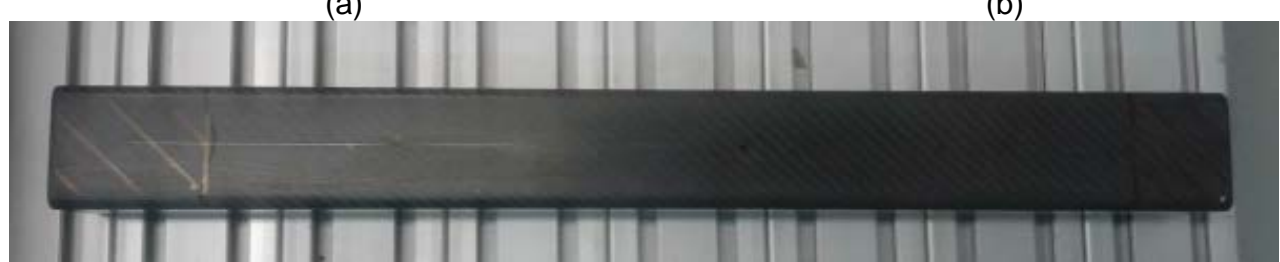

(c)

Fig. 4. Aeronautical components inspected by IRT NDT and analysed with the PTD method Wing section made of CFRP with titanium leading edge reinforcement (a), piece of landing gear gate made of CFRP with nomex honeycomb core (b), and tail of UAV made of CFRP.

The visualization of the defects showed by the projected thermal diffusivity method is clearly different from the one provided by the raw inspection and the common processing algorithms (Thermographic Signal Reconstruction, FFT, PCT or High Order Statistics). The PTD method shows defects with strong variations in the spatial signal profile (figures $2 \mathrm{c}$ and $2 \mathrm{~d}$ ). This result may be interpreted as the projection onto the $X Y$ coordinate plane of the heat diffused into the object due to the applied stimulation (red arrows in figure 3). Thus, vertical heat diffusion results in a zero projection, areas close to the edges of a defect induce a higher lateral diffusion, which consequently results in a higher projection, and the edges of defects produce maximum values of projected values.

\subsection{Results in real components}

Real aeronautical components have been inspected and analysed with the PTD method as a next step in the development process of the algorithm. Optical step heating thermographic inspections were conducted at the laboratory using two halogen lamps of $1000 \mathrm{~W}$ at $80 \%$ capacity. The lamps illuminated the specimens for $10 \mathrm{~s}$ so the temperature increase and decay were recorded for 10 seconds each stage, making a total of 20 seconds for the whole inspection. Infrared images were acquired using a FLIR SC5500 model camera. This camera is equipped with a cooled Indium antimonide detector that operates in the $2.5-5.1 \mathrm{~mm}$ waveband with $20 \mathrm{mK}$ of thermal sensitivity. Although the camera has a maximum frame rate of $383 \mathrm{~Hz}$ at full-frame, the experiments were recorded at $50 \mathrm{~Hz}$ to reduce the size of acquired videos.

The first component inspected with the aforementioned methodology was a section of leading edge made of carbon fibre reinforced polymer (CFRP) with the front zone reinforced with a titanium plate (figure 4a). Both parts, the CFRP structure and the titanium plate, were manufactured separately and eventually joined together with adhesive. This gluing process is complex and sometimes small debonded areas appear. The detection and growth control of these defects is essential to ensure the integrity of the wing and the whole plane. The inspections conducted on this specimen showed the presence of several debonded areas. Some of these results are shown in figure 5. It may be observed that the PTD analysis provides a high improvement in the visualization of the defects. Moreover, the spatial profiles produced by the PTD method indicate the exact area occupied by the debonding, enabling quick measures and comparisons with the inspection history. 


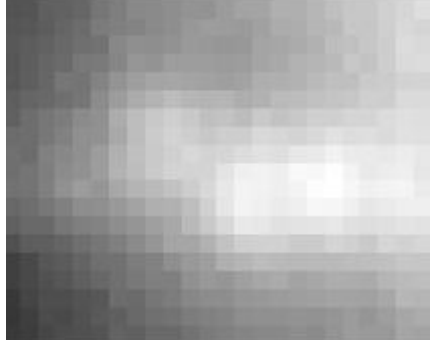

(a)

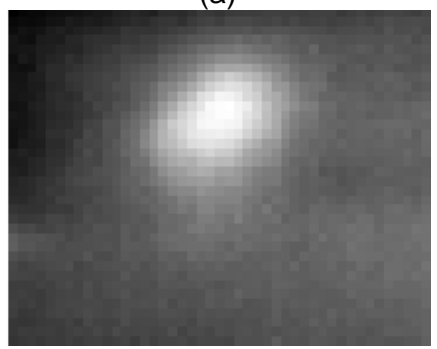

(d)

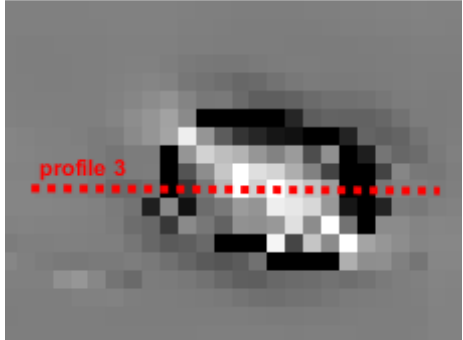

(b)

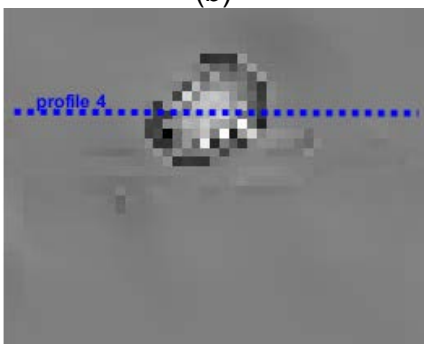

(e)

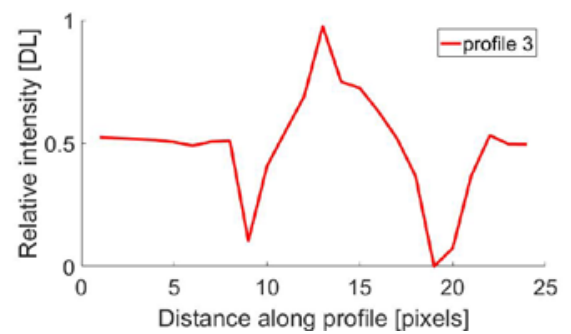

(c)

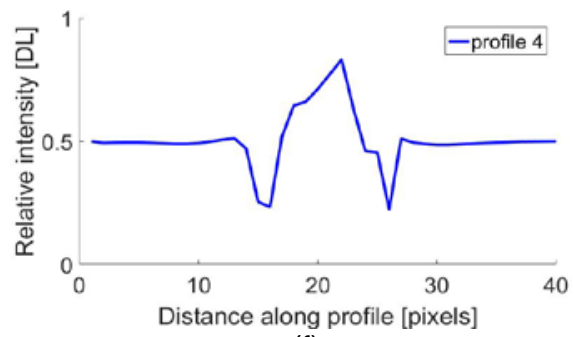

(f)

Fig. 5. Debonding defects detected in the inspections on the leading edge of the wing section. Raw data $(a, d)$, PTD analysis $(b, e)$ and spatial profile of PTD analysis $(c, f)$.

Two additional specimens were inspected in the tests campaign. The first one consisted in a UAV tail with square-section tubular shape made of CFRP (figure 4c). It had monolithic structure and the low thickness of the tube made it prone to undergo delaminations due to low energy impacts. Therefore impacts were applied to this specimen to induce delaminations in a controlled way. The impact energies were 5, 10 and 15 Joules applied with a homologated impactor system. The other specimen consisted in a piece of landing gear gate of an airplane (figure 4b). It was manufactured in a complex sandwich structure of different thicknesses. The external plies were manufactured in CFRP and the core in nomex honeycomb. This component is prone to receive impacts of small stones and gravel in the runway during the take-off and landing. Therefore impacts of low and medium energy levels were applied to this component in order to induce delaminations. Energies ranged from 35 to 120 Joules were applied to this specimen with the same impactor system as in the previous case.

The results obtained in the inspections on these specimens showed the different severities of the delaminations produced by the impacts, enabling to identify the areas affected with high accuracy. In general, delaminations produced by low energy levels showed two separated areas of damage around the impact point (figure 6b), while higher energy levels applied to the thicker specimen showed only one affected area just in the impact point (figure 6e). The PTD method provided improved visualization of defects with clear identification of the limits of damaged areas.

In order to assess the improvement provided by the PTD method, the SNR metric was used to objectively measure the level of the signal-to-noise ratio of the detected indications. The calculation of the SNR is based on the definition of two areas: a defective area, which encloses points from the IR image inside a detected indication, and a reference area, which encloses points outside the detected indication and is located close to the defect in order to be subject to the same external conditions. The SNR metric for a defect is calculated as shown in equation (5), where Def $\mu$ is the arithmetic mean of all the pixels inside the defective area, Ref $\mu$ is the arithmetic mean of all the pixels inside the reference area, and Refo is the standard deviation of the pixels inside the reference area. The SNR values obtained for the analysed defects and conventional processing techniques are shown in table 1 . The analysis capacity of the PTD is verified.

$$
S N R=20 \log _{10}\left(\frac{\left|D e f_{\mu}-\operatorname{Re} f_{\mu}\right|}{\operatorname{Re} f_{\sigma}}\right)
$$

Table 1. SNR values calculated for the analysed defects and different processing techniques including PTD analysis.

\begin{tabular}{|c|c|c|c|c|c|c|c|}
\hline PROCESSING & DEF2 & DEF3 & DEF4 & DEF5 & DEF6 & DEF7 & DEF8 \\
\hline RAW & 35,32 & 39,74 & 38,83 & 18,61 & 18,71 & 30,62 & 30,59 \\
\hline TSR & 33,71 & 40,95 & 38,48 & 15,59 & 5,93 & 30,29 & 28,05 \\
\hline DER1 & 38,43 & 48,91 & 49,01 & 22,89 & 21,64 & 38,94 & 41,55 \\
\hline DER2 & 34,51 & 37,49 & 42,92 & 22,05 & 17,62 & 31,83 & 37,09 \\
\hline FFT & 38,10 & 29,35 & 31,31 & 14,23 & 7,63 & 20,66 & 34,97 \\
\hline PCA & 29,71 & 36,23 & 38,27 & 21,56 & 24,63 & 23,05 & 37,66 \\
\hline PTD & $\mathbf{5 4 , 2 0}$ & $\mathbf{5 4 , 4 8}$ & $\mathbf{5 7 , 2 0}$ & $\mathbf{2 4 , 4 7}$ & $\mathbf{2 7 , 9 8}$ & $\mathbf{4 0 , 0 7}$ & $\mathbf{4 4 , 3 3}$ \\
\hline
\end{tabular}




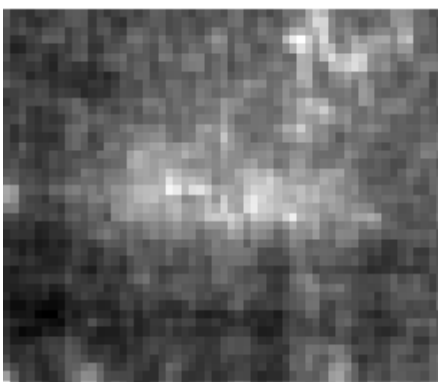

(a)

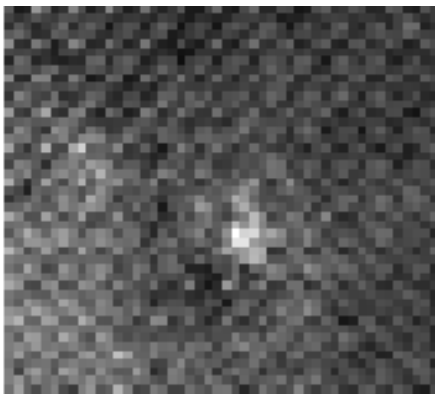

(d)

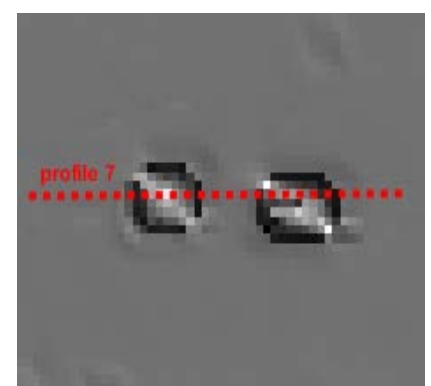

(b)

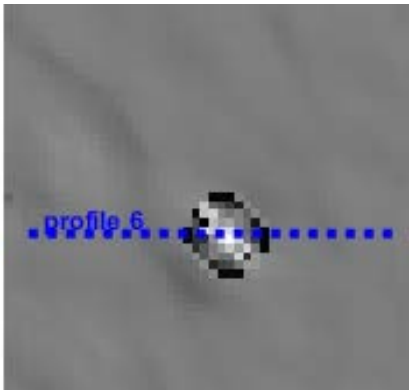

(e)

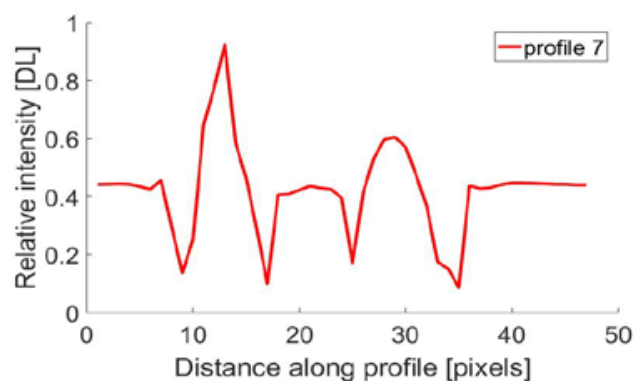

(c)

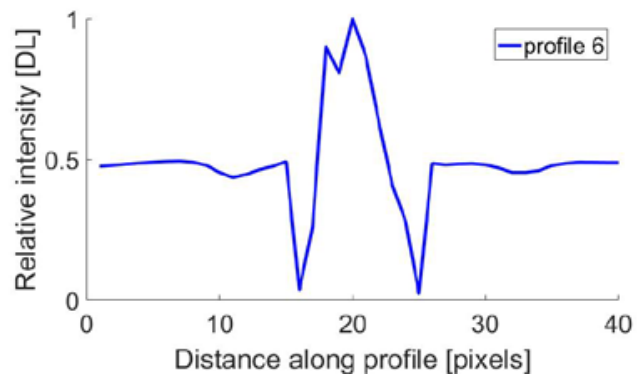

(f)

Fig. 6. Delamination defects detected in the inspections on the UAV tail $(a, b, c)$ and on the piece of landing gear gate $(d, e, f)$. raw data $(a, d)$, PTD analysis $(b, e)$ and spatial profile of PTD analysis $(c, f)$.

\section{Conclusions and future work}

The projected thermal diffusivity method has recently been developed for IRT NDT inspections. This method enables to analyse the behaviour of the thermal flow by the projection of the 3D heat diffusion equation onto a coordinate plane. The analysis capacities had been verified under computationally generated tests and experimental tests on calibrated samples. A physical interpretation of the results provided by the PTD method was proposed according to the capacity showed by the method for discriminating defects and the surrounding area affected by their presence. Moreover, it was experimentally proved that results obtained with the projection method increased the SNR of the initial temperature more than $15 \%$ and up to $50 \%$ by reducing the content of noise and reducing the irregularities in the stimulation process.

The PTD method has been applied in this study to real aeronautical components in order to validate the results and optimize its performance. It was demonstrated the high sensitivity of the PTD method to the noise content of the input data so that preliminary smoothing filtering is necessary for a correct application. However, the filtering stage reduces the accuracy in shape detection provided by the PTD method. Therefore an important task in future work will be to improve the relation between noise content and shape accuracy by considering alternative smoothing techniques.

The computational cost of the current PTD algorithm is high so limited areas can be analysed in each inspection with a standard computer. This issue has low influence on the development of the method; however, it becomes relevant when the objective is the application to on-site inspections where the time is a critical parameter. Therefore, next steps will deal with the optimization of the code produced for the PTD method in order to optimize the memory usage and computational operations.

The PTD method is still under development stage so further information related the heat diffusion inside the material is expected to be obtained. The results showed in this study correspond to the second defined category, i.e. finite thickness without presence of defects. The most general case, i.e. any thickness with presence of defects, is currently being solved. It was proved that the procedure applied to solve the cases of the second category is useless for those of the third category. Different inversion approaches are being considered to achieve this goal.

The method has been initially developed for optical step heating stimulation due to specific experimental requirements. The application to other types of stimulations is considered to have high practical interest but it needs to adapt the current procedure. Preliminary tests have been conducted with adjusted versions of the PTD method, not only for pulsed heating but also for step cooling down stimulations, producing results with characteristics similar to those showed in this study for step heating. 


\section{REFERENCES}

[1] Meola C, Carlomagno G.M. Recent advances in the use of infrared thermography, Meas Sci Technol. 2004;15(9)R27.

[2] Usamentiaga R, Venegas P, Guerediaga J, Vega L, López I. A quantitative comparison of stimulation and postprocessing thermographic inspection methods applied to aeronautical carbon fibre reinforced polymer. Quantitative InfraRed Thermography Journal. 2013;10(1)55-73.

[3] Rogalski A, Recent progress in infrared detector technologies, Infrared Phys Technol. 2011;54(3)136-154.

[4] Ibarra-Castanedo C, Gonzalez D, Klein M, Pilla M, Vallerand S, Maldague X. Infrared image processing and data analysis, Infrared Phys Technol 2004;46(1)75-83.

[5] Shepard S.M, Lhota J.R, Rubadeux B.A, Wang D, Ahmed T. Reconstruction and enhancement of active thermographic image sequences, Opt Eng. 2003;42(5)1337-1342.

[6] Venegas P, Perán J, Usamentiaga R, Sáez de Ocáriz I. Projected thermal diffusivity analysis for thermographic nondestructive inspections. International Journal of Thermal Sciences. 2018;124:251-262. 